\title{
Riesgo neurológico en el niño de mediano riesgo neonatal
}

\author{
Vericat $A,{ }^{1}$ Orden $\mathrm{AB}^{2}$
}

\section{Resumen}

INTRODUCCIÓN: los neonatos de mediano riesgo suelen presentar alteraciones del neurodesarrollo, sutiles o inaparentes, que pueden evidenciarse a mediano y a largo plazos; es por ello que se les considera niños de riesgo neurológico. La identificación de los factores de riesgo asociados con los problemas del neurodesarrollo es un primer paso para prevenir o reducir sus efectos.

OBJETIVO: con esta revisión cualitativa de la literatura especializada pretendemos describir las características de un grupo de neonatos denominados colectivamente recién nacidos de mediano riesgo neonatal y que, al nacer, no presentan compromiso evidente del desarrollo neurológico. Indagamos, también sobre los factores etiológicos asociados con esta condición.

MATERIALES Y MÉTODOS: realizamos una búsqueda bibliográfica en las bases Scientific Electronic Library Online (SciELO), Literatura Latinoamericana y del Caribe en Ciencias de la Salud (LILACS), la Biblioteca Nacional de Medicina de Estados Unidos de Norte América (NLM-Medline) y la Red de Revistas Científicas de América Latina y el Caribe, España y Portugal (RedALyC) centrada en factores de riesgo neurológico.

RESULTADOS: el incremento de los controles prenatales, la reducción de los nacimientos pretérmino y del parto por cesárea son factores relevantes para el óptimo neurodesarrollo. Luego del parto es necesario extremar los cuidados del neonato en las Unidades de Cuidado Intensivo Neonatal controlando cuadros tales como la hiperbilirrubinemia, la sepsis neonatal y la dificultad respiratoria, asociados con alteraciones del desarrollo en los neonatos de mediano riesgo.

CONCLUSIONES: las intervenciones para evitar o reducir el riesgo neurológico en los neonatos de mediano riesgo deben establecerse desde el inicio de la gestación y continuar durante toda la permanencia en las Unidades de Cuidado Intensivo Neonatal.

PALABRAS CLAVE: recién nacido, trastornos del neurodesarrollo, factores de riesgo, neonatología, desarrollo infantil.
${ }^{1}$ Pediatra especialista en Neurodesarrollo. Dra en Medicina. Cátedra de Pediatría B de la Universidad Nacional de La Plata. Argentina

${ }^{2}$ Doctora en Ciencias Naturales. Investigadora Independiente del Consejo Nacional de Investigaciones Científicas y técnicas (Conicet). Instituto de Desarrollo en Investigaciones Pediátricas (IDIP MS/CIC/PBA) Hospital Sor María Ludovica de La Plata. Argentina.

Hospital Sor María Ludovica de La Plata, Argentina.

Recibido: 19 de noviembre del 2016

Aceptado: 26 de abril del 2017

Correspondencia

Agustina Vericat

agustinavericat@gmail.com

Este artículo debe citarse como Vericat A, Orden AB. Riesgo neurológico en el niño de mediano riesgo neonatal. Acta Pediatr Mex. 2017;38(4):255-266. 


\title{
Neurological risk in children of moderate neonatal risk.
}

\author{
Vericat $A,{ }^{1}$ Orden $A B^{2}$
}

\begin{abstract}
INTRODUCTION: Moderate-risk neonates (MRN) may have subtle neurodevelopmental disorders, which can be evident in the medium and long term. That is why they are considered children with neurological risk. Identifying risk factors associated with neurodevelopmental disorders is a first step in preventing or reducing their effects.
\end{abstract}

OBJECTIVE: To describe the characteristics of a group of newborns collectively referred to as moderate-risk neonates, who at birth do not show neurodevelopmental disorders, and to investigate the etiological factors associated with this condition.

METHODS: We carried a bibliographic search was performed in the SciELO, LILACS, Medline and RedALyC databases focused on neurological risk factors.

RESULTS: Increased prenatal control and the reduction of preterm and cesarean delivery are important factors for optimal neurodevelopment. After delivery, newborn care in NICUs is critical, by controlling pathologies such as hyperbilirubinemia, neonatal sepsis, and respiratory distress associated with developmental abnormalities in MRN.

CONCLUSIONS: Interventions to avoid or reduce neurological risk in MRN must be established from the beginning of pregnancy and continued during the NICU stay.

KEYWORDS: newborn; risk factors; neurodevelopmental disorders; neonatology; child development

\begin{abstract}
${ }^{1}$ Pediatra especialista en Neurodesarrollo. Dra en Medicina. Cátedra de Pediatría B de la Universidad Nacional de La Plata. Argentina

${ }^{2}$ Doctora en Ciencias Naturales. Investigadora Independiente del Consejo Nacional de Investigaciones Científicas y técnicas (Conicet). Instituto de Desarrollo en Investigaciones Pediátricas (IDIP MS/CIC/PBA). Hospital Sor María Ludovica de La Plata. Argentina.
\end{abstract}

Hospital Sor María Ludovica de La Plata, Argentina.

Correspondence

Agustina Vericat

agustinavericat@gmail.com

\section{INTRODUCCIÓN}

Los recién nacidos de riesgo son una población de niños que presentan determinadas características perinatales (factores de riesgo) entre los cuales figuran prematurez, bajo peso al nacer, infecciones, asfixia, cardiopatías congénitas, entre otras, que los exponen a adquirir una enfermedad o a morir. ${ }^{1}$ Una forma de clasificarlos es de acuerdo con el órgano o sistema afectado por el efecto adverso; por ejemplo, los pacientes que recibieron medicación ototóxica pueden presentar mayor riesgo sensorial, mientras que los niños nacidos en posición podálica serán más susceptibles de luxación de cadera. De este modo, los recién nacidos son colectivamente referidos como de riesgo sensorial, cardiovascular o neonatos de riesgo neurológico, entre 
otros. Otra forma de clasificar a los neonatos se relaciona con la magnitud del riesgo: alto, moderado y bajo. Los neonatos de alto riesgo neonatal se caracterizan por presentar altas morbilidad y mortalidad y requerimientos de cuidados específicos: tienen mayor probabilidad de presentar trastornos, fundamentalmente sensoriales y del neurodesarrollo durante la infancia. ${ }^{2}$ En el extremo opuesto, los niños de bajo riesgo neonatal son recién nacidos que por sus características son aparentemente sanos: recién nacidos de término, sin antecedentes de gravedad en su historia familiar, gestacional y perinatal y con examen físico y adaptación al medio extrauterino adecuados., ${ }^{3,4}$ Mediando entre ambos extremos se encuentra un grupo denominado neonatos de moderado o mediano riesgo; se estima que $12 \%$ de los embarazos darán lugar a recién nacidos de riesgo moderado. ${ }^{5}$ Entre sus principales características la población de neonatos de mediano riesgo tiene bajas tasas de mortalidad pero altas tasas de morbilidad; son niños que presentan enfermedades variadas que en general no ponen en riesgo su vida y se resuelven rápidamente, aunque al nacer requieren atención en una Unidad de Cuidados Intensivos Neonatales. Es una población heterogénea, integrada fundamentalmente por dos tipos de paciente: los prematuros tardíos (late preterms) y los niños nacidos de término con patologías de escasa gravedad como problemas respiratorios, hiperbilirrubinemia, infecciones perinatales, etcétera. Las características comunes a todos ellos son requerimiento de cuidados especiales al nacer, mayor morbilidad que la población "normal", mayores secuelas a largo plazo y mayor prevalencia de problemas en el neurodesarrollo.

El incremento de alteraciones del desarrollo en los niños ingresados en las Unidades de Cuidados Intensivos Neonatales, así como de las secuelas a corto o largo plazo, resulta paradójicamente de una marcada disminución de la mortalidad debida a los importantes adelantos tecnológicos de estas unidades. ${ }^{6}$ Debido a que los neonatos de mediano riesgo pueden presentar trastornos inaparentes al alta, tienden a ser subestimados en cuanto a sus posibles secuelas a mediano y largo plazos. Sin embargo, hay evidencia de que estos niños tienen problemas del neurodesarrollo mayores a los de la población general. ${ }^{1}$ Realizamos una búsqueda bibliográfica en las bases de datos de SciELO, LILACS, Medline, y RedALyC centrada en factores de riesgo neurológico con el objetivo de describir las características de este grupo de neonatos de mediano riesgo.

\section{NIÑOS DE MEDIANO RIESGO NEONATAL}

Los niños de mediano riesgo neonatal pueden presentar alteraciones del neurodesarrollo por diferentes mecanismos. Muchos de estos son prevenibles a través de un embarazo controlado, mientras otros (no prevenibles) pueden ser tratables en forma oportuna a fin de minimizar los posibles daños. A continuación se describen los principales factores de riesgo en los niños de mediano riesgo nenonatal.

\section{Factores pre y perinatales}

Hipotiroidismo congénito y diabetes gestacional: el hipotiroidismo congénito es una insuficiencia tiroidea debido a la ausencia total o parcial de la glándula tiroidea o a la falta de acción de hormonas tiroideas durante la vida fetal. ${ }^{7}$ Cuando se manifiesta desde la etapa fetal temprana afecta principalmente el desarrollo del sistema nervioso central y esquelético. Es la enfermedad endocrinológica congénita más frecuente, con una prevalencia aproximada de 1 por cada 4,000 recién nacidos vivos, y la causa más común de retardo mental prevenible por medio de un diagnóstico y tratamiento tempranos. ${ }^{8} \mathrm{~A}$ veces los niños pueden tener signos y síntomas como parto prolongado y laborioso (como el feto es hipotónico no recorre fácilmente el canal del parto), recién nacido hipotérmico o con hipotermia transitoria, mixedema, fontanela posterior amplia 
(> $5 \mathrm{~cm}$ ), distensión abdominal, constipación, ictericia prolongada ( $>3$ días), piel seca, hernia umbilical, Ilanto ronco, escaso incremento ponderal y retardo del crecimiento lineal.

En el caso de la diabetes gestacional es conocido que los hijos de madres diabéticas suelen ser macrosómicos. Un 30\% de los recién nacidos pueden presentar peso mayor a 4,000 g o mayor al percentil 90 para su edad. A consecuencia de su mayor tamaño los traumatismos obstétricos son más frecuentes; también pueden presentar hipoglucemia, hipocalcemia e hipomagnesemia que requieren tratamiento inmediato ya que pueden causar letargia, tetania, coma, convulsiones, apneas o shock, pudiendo alterar el neurodesarrollo en la infancia. ${ }^{9}$

Infecciones prenatales: los trastornos del desarroIlo han sido vinculados con diversas infecciones prenatales. El Tripanosoma cruzi, vector de la enfermedad de Chagas, puede ser trasmitido al feto ocasionando Chagas congénito, cuya prevalencia en países endémicos fluctúa entre 60 y $90 \% .{ }^{10}$ Si bien se ha referido que entre 30 y $50 \%$ de los casos neonatales presentan alteraciones del sistema nervioso central, ${ }^{11}$ otros estudios no han hallado manifestaciones clínicas de meningoencefalitis, ${ }^{12}$ aunque se ha informado que hasta un $30 \%$ de los pacientes puede presentar calcificaciones intracraneales a largo plazo, posiblemente producidas por invasión parasitaria sintomática al inicio de la enfermedad. ${ }^{13}$

Otra enfermedad perinatal que presentan los neonatos de mediano riesgo, de relevancia por las secuelas que produce a nivel del sistema nervioso, es la toxoplasmosis humana, producida por el Toxoplasma gondii. Los efectos de importancia clínica ocurren en los recién nacidos con la infección adquirida intraútero o en pacientes inmunodeprimidos. Aunque la mayoría de los niños son asintomáticos al nacer algunos estudios han mostrado que sin terapia adecuada más de
$70 \%$ puede desarrollar microcefalia, hidrocefalia, calcificaciones cerebrales y coriorretinitis, llevando a visión borrosa y pérdida o dificultad de la visión central, entre otros signos y síntomas. Actualmente se conoce que $50 \%$ de los niños sufrirán daños neurológicos a largo plazo. ${ }^{14}$

La sífilis en su forma congénita afecta aproximadamente a $10 \%$ de los recién nacidos vivos en diferentes países. ${ }^{15}$ Esta patología puede producir prematurez, retardo del crecimiento intrauterino, síndrome nefrótico, neumonitis, hepatoesplenomegalia, conjuntivitis, adenomegalias y anemia hemolítica, entre otras. En 50\% de los recién nacidos con esta condición los síntomas no son inmediatos sino que la infección se expresa semanas o meses más tarde. Así, algunos signos neurológicos de sífilis tardía tales como retraso mental, hidrocefalia y sordera neurogénica ${ }^{15}$ pueden aparecer a partir de los 2 años de vida.

Los niños infectados por el virus de la inmunodeficiencia humana con síntomas de la enfermedad, presentan cuadros infecciosos repetitivos, hepatoesplenomegalia y alteraciones en las habilidades motoras, de integración viso-motora y percepción viso-espacial y, en menor grado, del lenguaje. La infección por por el virus de la inmunodeficiencia humana se asocia con un mayor riesgo de daño al sistema nervioso central, considerándose que entre 15 y $25 \%$ de los niños podían presentar un daño grave $y$, a menudo, manifestaciones progresivas de lesión. ${ }^{16}$ Se ha informado que aquellos niños con deficiencias cognoscitivas tempranas y con retraso o deterioro motor tienen pérdida de múltiples funciones del desarrollo. ${ }^{17} \mathrm{No}$ obstante, la frecuencia con la cual se presentan las manifestaciones neurológicas en estos grupos de pacientes es variable. Un estudio colaborativo europeo reportó una frecuencia de signos neurológicos severos en $13 \%$ de los niños infectados. Esta cifra se elevó a $31 \%$ en niños con diagnóstico de SIDA. ${ }^{18}$ Sin el tratamiento 
antirretroviral, la encefalopatía por el virus de la inmunodeficiencia humana se presenta en general antes del año de vida.

Retardo del crecimiento intrauterino: las condiciones adversas durante la vida fetal han sido relacionadas con cambios estructurales y funcionales en el neurodesarrollo desde el período neonatal hasta la adolescencia. Los niños con retardo del crecimiento intrauterino suelen presentar asfixia perinatal, aspiración de meconio, hipocalcemia, hipotermia, policitemia, malformaciones congénitas e infecciones. ${ }^{19}$ Además de la morbilidad neonatal, el retardo puede producir alteraciones del desarrollo neuropsíquico; tanto el simétrico como el asimétrico puede alterar el neurodesarrollo. En general, existe peor pronóstico en los casos de retardo del crecimiento intrauterino simétrico, ligado a injurias durante la gestación temprana. Sin embargo, muchos eventos asociados con el neurodesarroIlo ocurren en la gestación tardía. Entre estos se incluyen: proliferación glial, migración neuronal, diferenciación y organización cortical y conectividad entre distintas estructuras cerebrales. ${ }^{20}$ Así, los neonatos con retardo del crecimiento intrauterino pueden presentar tanto secuelas sutiles o bien anormalidades más importantes del neurodesarrollo. Los problemas más observados en estos niños incluyen tanto disfunciones de la motricidad fina y gruesa, del comportamiento individual (déficit de atención, hiperactividad) y social, y a nivel cognitivo. Al respecto se ha informado que niños de edad escolar, nacidos pequeños para la edad gestacional con retardo del crecimiento intrauterino moderado, tuvieron mayor riesgo de dificultad cognitiva (OR: 1.87, 95\% Cl: 1.24-2.82) y del comportamiento (OR: $1.66,95 \% \mathrm{Cl}: 1.04-2.62)$ que los adecuados para la edad gestacional. La relación entre la función cognitiva y el retardo del crecimiento intrauterino también ha sido estudiada en adultos nacidos con dicho retardo, que alcanzaron puntuaciones IQ significativamente menores que sus pares sin retardo del crecimiento intrauterino (diferencia media -6.3). ${ }^{21}$

Asfixia perinatal: la asfixia perinatal es una de las principales causas de morbilidad y mortalidad perinatales. La lesión cerebral por asfixia perinatal se debe a la hipoxia e isquemia cerebral que finalmente llevan a disfunción cerebral y muerte neuronal. Su incidencia varía con la edad gestacional, siendo en el recién nacido a término de $0.5 \%$, mientras que en el recién nacido de menos de 30 semanas la cifra se eleva a $50 \%{ }^{22}$ Las manifestaciones a largo plazo se presentan como secuelas motoras (con diversas formas de parálisis cerebral), cognitivas, epilepsia y déficits neurosensoriales. También la intensidad y duración de la sintomatología clínica tienen valor pronóstico, principalmente si se presentaron convulsiones o coma. Se ha descripto la asociación entre puntaje de Apgar y trastornos del neurodesarrollo, ${ }^{23}$ y también entre un bajo puntaje Apgar y déficit atencional e hiperactividad en niños de edad escolar. ${ }^{24}$ Aunque la puntuación de Apgar menor a 7 es un índice fiable de la condición del recién nacido inmediatamente después del nacimiento, la asociación entre baja puntuación Apgar y discapacidad neurológica no predice qué alteraciones neurológicas pueden ocurrir a largo plazo. ${ }^{25}$

\section{Parto}

Parto por cesárea: se ha sostenido que el estrés del trabajo de parto es uno de los factores que provocan hemorragia intraventricular $y$ leucomalacia periventricular, fuertemente correlacionadas con el neurodesarrollo. ${ }^{26}$ El cráneo fácilmente deformable, sobre todo en el bebé prematuro, puede elevar la presión venosa durante el parto vaginal, Ilevando a la idea que el parto por cesárea podría reducir estas complicaciones. No obstante, la evidencia parece indicar que la evitación del parto vaginal no confiere ninguna ventaja para el feto prematuro con pre- 
sentación cefálica, ni jugar un rol significativo en el deterioro del neurodesarrollo en neonatos de bajo y muy bajo peso al nacer. ${ }^{27}$ De hecho, el parto por cesárea es causa de mayor morbilidad que el parto vaginal, aún en los embarazos de bajo riesgo. ${ }^{28}$ Sin embargo, los efectos de la cesárea sobre el desarrollo psicomotor humano han sido muy poco explorados. En modelos animales se han observado deterioros en la memoria espacial y la conducta, así como alteraciones de la morfología dendrítica y de distintas estructuras del sistema nervioso central, ${ }^{29,30}$ indicando que procesos similares puedan ocurrir en el cerebro del feto humano. Al respecto, se han informado asociaciones entre parto por cesárea y desarrollo del lenguaje y de la motricidad fina, en niños que al alta eran aparentemente sanos. ${ }^{1,31}$ En niños de mayor edad se halló que la cesárea puede producir dificultades del aprendizaje, dislexia, dispraxia y déficit de atención. ${ }^{32}$

\section{Factores postnatales}

Prematurez tardía: aproximadamente $12 \%$ de los nacimientos corresponden a niños prematuros..$^{33}$ De éstos, 70 a $75 \%$ son prematuros tardíos, ${ }^{34}$ definidos como aquellos niños nacidos entre las 34 y 37 semanas de gestación. Estos prematuros muestran un mayor riesgo de presentar retardo del desarrollo físico y de trastornos sutiles del desarrollo, retrasos del desarrollo, parálisis cerebral, retardo mental y convulsiones en la niñez temprana respecto a los niños nacidos a término. ${ }^{35}$ La probabilidad de que un prematuro tardío desarrolle al menos una complicación médica es cuatro veces mayor que la de un niño a término. ${ }^{36}$ Esto incluye complicaciones a largo plazo del desarrollo psicomotor y cognitivo tales como retrasos del desarrollo, fracaso escolar, trastornos del comportamiento y discapacidades sociales. ${ }^{37} \mathrm{En}$ efecto, se ha informado que el riesgo de requerir educación especial aumenta con la menor edad gestacional, incluso en los nacidos con 33 a 36 semanas (OR 1.53, 95\% Cl: 1.43-1.63). ${ }^{32}$
Dificultad respiratoria: la dificultad respiratoria del neonato es la causa más frecuente de morbilidad y mortalidad neonatales. ${ }^{38}$ Su forma más leve, denominada dificultad transitoria del recién nacido, tiene una prevalencia de $37 \%$ y se manifiesta por aumento de la frecuencia respiratoria y retracciones torácicas leves desde el nacimiento. Si bien puede producir cuadros clínicos severos tales como convulsiones, encefalopatía hipóxico isquémica, hemorragias intraventriculares y atrofia cerebral; ${ }^{39}$ también puede asociarse con trastornos sutiles como retrasos del lenguaje. ${ }^{1}$ Junto a la depresión al nacer (bajo puntaje de Apgar) la dificultad respiratoria ha sido considerada como factor de riesgo del neurodesarrollo en la primera infancia. ${ }^{40}$

Hiperbilirrubinemia: este trastorno es una de las diez primeras causas de morbilidad neonatal en las Unidades de Cuidados Intensivos Neonatales. En la población general la hiperbilirrubinemia neonatal alcanza aproximadamente 20\%. ${ }^{41}$ Esta cifra aumenta en los neonatos de pretérmino, en los cuales alrededor de $80 \%$ puede desarrollar algún grado de ictericia. ${ }^{42}$ Los efectos en el sistema nervioso son principalmente la hipoacusia neurosensorial y, en casos severos, puede producir encefalopatía neonatal bilirrubínica (Kernicterus). En los neonatos de mediano riesgo se han reportado secuelas a largo plazo que incluyen retrasos del desarrollo, dificultades escolares y, en casos más severos, retraso mental. ${ }^{43}$ Aunque la bilirrubina sérica debe ser elevada para producir daño neurológico los estudios no son concluyentes. ${ }^{1,44}$ También se ha observado que sus efectos pueden ser reversibles; ${ }^{45}$ así, la hiperbilirrubinemia sería un factor necesario pero no suficiente para explicar el daño neurológico que se produce en algunos recién nacidos.

Sepsis del recién nacido: en países desarrollados la incidencia de sepsis neonatal alcanza entre 0.6 y $1.2 \%$ de los nacidos vivos, pero en países en desarrollo esta cifra puede incrementarse 
entre 20 y $40 \% .{ }^{46}$ Las sepsis graves ocurridas antes de los 6 meses de edad se asocian con trastornos sensoriales o motores en la infancia. Además de los daños propios de la sepsis muchos de los antibióticos utilizados para su tratamiento son ototóxicos, como algunos aminoglucósidos de uso frecuente en neonatología, pudiendo producir hipoacusias de diverso grado. ${ }^{47}$

\section{CUIDADOS, SEGUIMIENTO Y ALTA}

Muchos de los factores mencionados conducen a la internación del neonato en Unidades de Cuidado Intensivo Neonatal. Una vez que esto ocurre es necesario crear un ambiente con mínimo estrés para favorecer su desarrollo de una manera similar a la vida intrauterina. Estos cuidados han sido Ilamados "Cuidados Centrados en el Desarrollo" y su objetivo es favorecer el neurodesarrollo del niño. La pionera en este tipo de acciones fue Heidelise Als en la década de 1980, cuando creó el Programa de Evaluación y Atención Individualizada y Orientada al Desarrollo Neonatal (Neonatal Individualized Developmental Care and Assessment Program). ${ }^{48}$ Este programa capacita a los profesionales sanitarios para que controlen los estímulos externos capaces de causar daño al neonato: la luz, ruido, un mal manejo del dolor, el control de la temperatura, adecuada concentración de oxígeno, el posicionamiento y el método canguro. Un pilar fundamental de este programa es la implicación de los padres para el cuidado de sus hijos.

Una vez egresados de neonatología, los neonatos de mediano riesgo "aparentemente sanos" requerirán cuidados especiales y valoraciones del neurodesarrollo en forma periódica y estandarizada debido a su riesgo neurológico. Es por esto que en el desarrollo de su práctica médica el neonatólogo muchas veces debe tomar decisiones difíciles en relación con las conductas a seguir luego del alta: puede derivar al niño para un seguimiento neurológico exhaustivo, reali- zado en hospitales de mayor complejidad, en donde se somete al niño a una gran variedad de estudios diagnósticos o permitir su seguimiento en centros de atención primaria, en donde se realizarán evaluaciones clínicas periódicas a cargo del pediatra de cabecera, determinando cuándo es necesario que intervenga un especialista (neuropediatra kinesiólogo, terapista ocupacional, entre otros). En el primer caso, muchas familias deberán movilizarse hasta centros de mayor complejidad (como los hospitales pediátricos) requiriendo una atención especializada que tal vez no necesiten e insumiendo costos innecesarios. En el segundo, cabrá la posibilidad de que el pediatra de atención primaria pase por alto alguna patología específica de difícil diagnóstico o que su derivación sea tardía. Esta dificultad puede sortearse empleando pruebas de pesquisa neonatal en forma protocolizada, en momentos específicos de la consulta. Estas pruebas permiten realizar un rápido tamizaje del neurodesarrollo a fin de determinar qué pacientes deben ser derivados a un especialista en forma relativamente simple y objetiva. ${ }^{49} \mathrm{La}$ evaluación o pesquisa del desarrollo realizada en forma sistematizada en estos pacientes permite detectar, en forma oportuna, los posibles trastornos del neurodesarrollo e iniciar su tratamiento a corto plazo..$^{50}$

\section{DISCUSIÓN}

Dado el carácter multifactorial del retraso del desarrollo ${ }^{51}$ es necesario identificar aquellos factores posibles de intervención. Es bien conocida la necesidad de realizar cuidados prenatales de carácter preventivo, a través del seguimiento adecuado del embarazo por parte del equipo de salud, pero también se trabaja cada vez más en relación con los cuidados del niño una vez ingresado en la Unidad de Cuidado Intensivo Neonatal, mediante los Ilamados "cuidados centrados en el desarrollo", cuyo objetivo principal es la protección del cerebro. Si bien estas prác- 
ticas son aceptadas e implementadas en muchas Unidades de Cuidado Intensivo Neonatal aún es discutida su efectividad sobre el neurodesarrollo a largo plazo. ${ }^{52,53}$ Una cuestión central es hasta qué punto es posible reducir la morbilidad neurológica en los neonatos de mediano riesgo. Parecería que aún se está lejos de lograr este objetivo puesto que los factores de riesgo no siempre son adecuadamente controlados.

Se podrían delinear dos grandes etapas o ventanas de intervención: prenatal y postnatal. La intervención prenatal implica controlar o minimizar los factores que afecten potencialmente al neurodesarrollo: retardo del crecimiento intrauterino, prematurez y los relacionados con patologías maternas y del recién nacido, a fin de disminuir las posibles secuelas a corto y largo plazos. El retardo del crecimiento intrauterino produce hipoflujo placentario y, por ende, disminución del flujo cerebral. Las estrategias preventivas deben por lo tanto focalizar factores de riesgo maternos (adolescencia, edad avanzada, déficit nutricional, obesidad, hipertensión y pre-eclampsia, adicciones e infecciones), fetales (malformaciones genéticas y cromosómicas, infecciones, embarazo múltiple) y placentarios (placenta pequeña, placenta previa, anomalías vasculares o del cordón fetal, trombosis, desprendimiento, sangrado, trombofilia y restricción del flujo placentario). ${ }^{54,55} \mathrm{El}$ control de estos factores también redunda en la disminución de la prevalencia de la asfixia perinatal ya que, aunque durante mucho tiempo se atribuyó su la etiología a dificultades surgidas durante el momento del parto, ${ }^{56}$ actualmente se conoce que esto no es así y que es importante tener también en cuenta la desproporción feto pélvica e indicar la realización de monitoreos y ecografías de rutina. ${ }^{57}$

La prematurez conlleva los riesgos de un cerebro inmaduro en un medio no fisiológico (la Unidad de Cuidado Intensivo Neonatal) con los posibles daños secundarios al estrés, a maniobras intem- pestivas, a infecciones intrahospitalarias, etc. Entre las estrategias existentes para su prevención se encuentra el uso de progesterona en la embarazada, la eliminación del hábito tabáquico, el cerclaje en mujeres con partos prematuros previos y cuello cervical corto, la reducción del número de embriones implantados en tratamientos de fertilización y la reducción de cesárea sin indicación médica. ${ }^{58}$

El monitoreo regular del crecimiento fetal, especialmente en el tercer trimestre de la gestación, se recomienda en estos embarazos de riesgo, así como en los casos de retardo del crecimiento intrauterino, preeclampsia y diabetes gestacional, entre otros..$^{59}$ En el caso de la diabetes materna, además del monitoreo fetal, la Asociación Americana de Diabetes recomienda un plan de nutrición individual con evaluación basada en historia clínica, exámenes de laboratorio, hábitos, preferencias y recursos destinados a la alimentación, control del aumento de peso y actividad física. ${ }^{60}$

Luego del nacimiento deberán diagnosticarse y tratarse precozmente las complicaciones de este cuadro en el neonato: hipoglucemias, lesiones del plexo braquial, entre otras. Hay otros factores que requieren prevención pre y postnatal. La prevalencia de sepsis de aparición temprana sigue siendo sustancial en la era de la prevención del streptococo beta-hemolítico del grupo b (GBS) y afecta desproporcionadamente a los neonatos prematuros. Se necesita la identificación de estrategias para prevenir los nacimientos prematuros para reducir la sepsis neonatal. ${ }^{61} \mathrm{EI}$ cribado prenatal de GBS podría prevenir una gran parte de los casos, mientras que la sepsis causada por otros organismos es más a menudo una enfermedad de la prematuridad como por ejemplo la infección por E. coli. ${ }^{62}$ Recientes estudios han mostrado que la inmunización materna es una estrategia viable para mejorar los resultados de salud de las madres y los ni- 
ños pequeños. ${ }^{63}$ No obstante, las estrategias no pueden ser globales si se tiene en cuenta que en países de mayor ingreso hay un creciente número de niños extremadamente prematuros con altas tasas de infección nosocomial por microorganismos multirresistentes en las Unidades de Cuidado Intensivo Neonatal. ${ }^{64}$

El incremento de nacimientos prematuros, especialmente los prematuros tardíos, está estrechamente relacionado con el notable aumento de cesáreas no médicamente justificadas y al incremento de embarazos múltiples resultantes de la fertilización asistida. ${ }^{6,65}$ La tendencia creciente al parto por cesárea es global y sus efectos inmediatos, como la dificultad respiratoria, han sido objeto de numerosos estudios; sin embargo, las posibles secuelas cerebrales de las cesáreas han recibido escasa atención, aunque cada vez es mayor el conocimiento de los efectos de este procedimiento sobre la maduración cerebral intrauterina que continúa hasta el final de la gestación y puede generar importantes, e incluso graves, morbilidades a largo plazo. Aún no hay datos prospectivos del efecto de la cesárea programada sobre el desarrollo infantil. ${ }^{20}$ Se ha estimado que es posible reducir en un $80 \%$ las cesáreas sin justificación médica, ${ }^{37,58}$ que es uno de los principales factores responsables de la alta prevalencia de prematuros tardíos, aunque esta cifra varía entre distintos países. Además, la edad gestacional en la que se realiza la cesárea es importante no sólo porque el nacimiento antes de las 39 semanas puede aumentar la morbilidad respiratoria, sino también por la importancia de la maduración cerebral en el medio intrauterino sobre el futuro neurodesarrollo. ${ }^{20}$

Otros posibles agentes productores de lesión neuronal en los neonatos de mediano riesgo son el exceso o déficit de metabolitos u hormonas tales como el aumento de bilirrubina indirecta o el déficit de hormona tiroidea. La hiperbilirrubinemia puede ser tratada reduciendo su concentración a través de la fototerapia o la exanguinotransfusión, siendo importante el conocimiento del grupo y factor de la madre y del niño y la realización de la prueba de Coombs. Por otra parte, el hipotiroidismo puede ser controlado con administración de hormona tiroidea en forma precoz, al detectar dicha enfermedad mediante el estudio de errores congénitos. ${ }^{66} \mathrm{Si}$ todos estos posibles "controles" fallan (embarazo controlado, parto supervisado, estadía en Unidades de Cuidado Intensivo Neonatal minimizando riesgos y cuidando el cerebro) se estará frente a un neonato de mediano riesgo. Este niño, al momento del alta, será un niño "aparentemente sano", pero que conllevará riesgo neurológico. El neonato de riesgo neurológico es definido como "aquel niño que por sus antecedentes pre, peri o postnatales tiene mayor probabilidad de manifestar problemas de desarrollo cognitivos, motores, sensoriales o de comportamiento en los primeros años de vida, pudiendo ser transitorios o definitivos". ${ }^{67}$ Presentan inicialmente un daño cerebral inaparente, con un período libre de síntomas que dependerá de los medios tecnológicos para detectarlos, seguido eventualmente de una O varias manifestaciones clínicas. ${ }^{68}$

Los niños que finalmente desarrollan síntomas pueden presentar dos tipos de secuelas neurológicas: aquellas que se expresan en el primer año de vida y que pueden ser leves, moderadas o graves; y otras más tardías, que se detectan en la edad escolar. Este último grupo se caracteriza generalmente por dificultades en el aprendizaje escolar, trastornos en la conducta, torpeza motriz, retraso del lenguaje o problemas en la adaptación social. ${ }^{67}$ Estas características más de tipo "cualitativo" se expresan fenotípicamente en una escala continua, donde los trastornos del neurodesarrollo se ubican en un espectro que transcurre de la normalidad a la patología. ${ }^{69}$ Será necesario entonces el seguimiento de estos neonatos desde el punto de vista del desarrollo, que como se mencionó comienza en un pri- 
mer nivel con la pesquisa del neurodesarrollo mediante diferentes escalas. Una vez realizada la pesquisa será necesario derivar al niño con resultados positivos a un especialista para su diagnóstico de certeza y para iniciar el tratamiento correspondiente. Eventualmente, el desarrollo ulterior del niño requerirá considerar otros factores que han sido reconocidos como protectores o estimulantes del desarrollo infantil, como por ejemplo la promoción de la lactancia materna, la alimentación adecuada, la educación materna y la estimulación temprana, entre otros.

\section{CONCLUSIONES}

La identificación de los factores de riesgo asociados con los problemas del neurodesarrollo es un primer paso para prevenir o reducir sus efectos. En este sentido las intervenciones deben establecerse desde el inicio de la gestación y continuar hasta el parto. El incremento de los controles prenatales, la reducción de los nacimientos pretérmino y del parto por cesárea parecen ser de fundamental importancia en este periodo. Luego del parto es necesario controlar los factores de riesgo neurológico, extremando los cuidados del neonato en la Unidad de Cuidados Intensivos Neonatales, en especial para aquel grupo de niños con trastornos inaparentes como los neonatos de mediano riesgo.

\section{REFERENCIAS}

1. Vericat A. Trastornos inaparentes del desarrollo psicomotor en niños de mediano riesgo neonatal egresados de una unidad de cuidados intensivos neonatales. Tesis Doctoral. Universidad Nacional de La Plata; 2015.

2. Silva CF, Leite AJ, Almeida NM, Leon AC, Olofin I, Rede Norte-Nordeste de Saúde Perinatal. Factors associated with neonatal death in high-risk infants: a multicenter study in High-Risk Neonatal Units in Northeast Brazil. Cad Saude Publica. 2014;30:355-68.

3. Gracia $\mathrm{S}$, Rite et al. Niveles asistenciales y recomendaciones de mínimos para la atención neonatal. Anales de Pediatría. Elsevier Doyma. 2013;1:11-51.

4. Doménech E, González N, Rodríguez-Alarcón J. Cuidados generales del recién nacido sano. En: Protocolos
Diagnósticos Terapéuticos de la Asociación Española de Pediatría. Madrid: Sociedad Española de Neonatología. 2008;19-28.

5. Coronel Rodríguez C, Merón de Cote MP. Seguimiento del recién nacido de riesgo. Pediatr Integral. 2006;10:425-36.

6. Ceriani Cernadas JM. Prematuros tardíos, un creciente desafío a corto y largo plazo Arch Argent Pediatr. 2015;113:482-4.

7. Bernal Carrasco J. Las hormonas tiroideas en el desarrollo del cerebro. En: Monografías de la Real Academia de Farmacia Monografía XXIX: Acción de las hormonas a nivel cerebral. Madrid; 2009;139-69.

8. Campos, MLP, Musso M, Keselman A, Gruñeiro L, Bergadá I, Chiesa A. Cognitive profiles of patients with early detected and treated congenital hypothyroidism. Arch Argent Pediatr. 2017;115:12-17.

9. Deshpande $S$, Ward Platt $M$. The investigation and management of neonatal hypoglycaemia. Semin Fetal Neonatal Med 2005;10:351-61.

10. Freilij $H$, Biancardi $M$, Lapeña A, Ballering $G$, Altcheh J. Enfermedad de Chagas congénita. Chagas en: El Siglo XXI. De la enfermedad a la problemática social. 2010:117-125.

11. Cucunubá ZM, Valencia-Hernández CA, Puerta CJ, SosaEstani, S Torrico F, Cortés JA, Muller EÁ (2014). Primer consenso colombiano sobre Chagas congénito y orientación clínica a mujeres en edad fértil con diagnóstico de Chagas. Infectio. 2014;18:50-65.

12. Campos-Valdez G, Canseco-Ávila LM, González-Noriega F, Alfaro-Zebadua O, Nava-Medecigo IY, Jiménez-Cardoso E. Maternal-fetal transmission of Trypanosoma cruzi, a health problem slightly studied in Mexico: case Chiapas. Salud Pública de México. 2016;58:378-384.

13. Sociedad Argentina de Cardiología. Consenso sobre Enfermedad de Chagas-Mazza. Buenos Aires: Consejo de Enfermedad de Chagas Dr Salvador Mazza, Sociedad Argentina de Cardiología. 2011:1-16.

14. López-Castillo CA, Díaz-Ramírez J, Gómez-Marín JE. Factores de riesgo en mujeres embarazadas infectadas por Toxoplasma gondii en Armenia-Colombia. Rev Salud Pública. 2005;7:180-90.

15. Ministerio de la Protección Social-Instituto Nacional de Salud de Colombia. Protocolo de sífilis congénita y gestacional. 2007:3-9.

16. Castro M, Martínez Y, González Velázquez J, Castillo I, Sánchez L. Evaluación neuropsicológica, factores psicosociales y comorbilidad psiquiátrica en pacientes pediátricos infectados con el VIH. Rev Chil Infectol. 2011;28:248-54.

17. Nicholson L, Chisenga M, Siame J, Kasonka L, Filteau, S. Growth and health outcomes at school age in HIV-exposed, uninfected Zambian children: follow-up of two cohorts studied in infancy. BMC pediatrics. 2015;15:66-70.

18. Figueroa Medrano LP, Ávila Figueroa C. Alteraciones del desarrollo neurológico en niños nacidos de madres con VIH. Perinatol Reprod Hum. 2004;18:149-55. 
Vericat A y Orden AL. Neurodesarrollo en neonatos de riesgo

19. Álvarez GL, Moreyra VI, Martínez MA, Mosqueda ED. Retardo del crecimiento intrauterino: diagnóstico. Rev Pos Vla Cát Med. 2005;48: 13-5.

20. Kapellou O. Effect of caesarean section on brain maturation. Acta Paediatr. 2011;100:1416-22.

21. Løhaugen GCC, Oslashstgård HF, Andreassen S, et al. Small for gestational age and intrauterine growth restriction decreases cognitive function in young adults. J Pediatr. 2013;163:447-53.

22. Sánchez-Zúñiga ME, Pérez Madero GC, Martín López $M L$, Pérez Moreno JC. Factores de riesgo y signos de alarma para daño neurológico en niños menores de un año de edad. Reporte de 307 casos. Rev Mex Neuroci. 2009;10:259-63.

23. Cruz Morales E, Guerra Labrada A, Barreras J. Evaluación del neurodesarrollo a los dos años de edad en niños y niñas que presentaron encefalopatía hipóxico isquémica durante la etapa neonatal. Duarzy. 2005;2:95-101.

24. Ehrenstein V, Pedersen L, Grijota M, Nielsen GL, Rothman KJ, Sørensen HT. Association of Apgar score at five minutes with long-term neurologic disability and cognitive function in a prevalence study of Danish conscripts. BMC Pregnancy Childbirth. 2009;2:9-14.

25. Buñuel Álvarez JC, Aparicio Sánchez JL. Un test de Apgar bajo a los cinco minutos se relaciona con mayor riesgo de trastorno por déficit de atención con hiperactividad. Evid Pediatr. 2011;7:68-9.

26. Ballabh P. Pathogenesis and prevention of intraventricular hemorrhage. Clinics in perinatology. 2014;47-67.

27. Wadhawan R, Vohr BR, Fanaroff AA, Perritt RL, Duara S, Stoll BJ, et al. Does labor influence neonatal and neurodevelopmental outcomes of extremely-low-birth-weight infants who are born by cesarean delivery? Am J Obstet Gynecol. 2003;189:501-06.

28. Ceriani Cernadas JM, Mariani J, Pardo A, Aguirre A, Pérez $C$, Brener $\mathrm{P}$, et al. Nacimiento por cesárea al término en embarazos de bajo riesgo: efectos sobre la morbilidad neonatal. Arch Argent Pediatr. 2010;108:17-23.

29. Simon-Areces J, Dietrich MO, Hermes G, Garcia-Segura LM, Arevalo MA, Horvath TL. Ucp2 Induced by Natural Birth Regulates Neuronal Differentiation of the Hippocampus and Related Adult Behavior. PLoS ONE 2012;8:1-12.

30. Juarez I, Gratton A, Flores G. Ontogeny of altered dendritic morphology in the rat prefrontal cortex, hippocampus, and nucleus accumbens following Cesarean delivery and birth anoxia. J Comp Neurol. 2008;507:1734-47.

31. Fraile Sánchez C. Posibles implicaciones del parto por cesárea en la aparición de problemas logopédicos. Tesis Doctoral. Universidad de Valadolid. 2015;2-74.

32. MacKay DF, Smith GC, Dobbie R, Pell JP. Gestational age at delivery and special educational need: retrospective cohort study of 407,503 schoolchildren. PLoS Med. 2010;7:2-10.

33. Hamilton BE, Martin JA, Ventura SJ. Births: preliminary data for 2005. National Vital Statistics Report. 2006;55:1-18.
34. Hurtado Suazo JA, Alvarez ME. El prematuro tardío. Hospital Materno Infantil. Granada, Bol. SPAO. 2009;31:151-7.

35. Petrini JR, Dias T, McCormick MC, Massolo ML, Green NS, Escobar GJ. Increased risk of adverse neurological development for late preterm infants. J Pediatr. 2009;154:169-76.

36. Arandia Valdez R, Ayala Balderrama M. Recién nacido "prematuro tardío" frente a los riesgos qué debe tenerse en cuenta. Gac Med Bol. 2010;33:64-9.

37. Vohr B. Long-term outcomes of moderately preterm, late preterm, and early term infants. Clin Perinatol. 2013;40:739-51.

38. Coto Cotallo GD, López Sastre J, Fernández Colomer B, Álvarez Caro F, Ibáñez Fernández A. Recién nacido a término con dificultad respiratoria: enfoque diagnóstico y terapéutico. En: Protocolos Diagnóstico Terapeúticos de la AEP. 2008. URL: http: //www.aeped.es/sites/default / files/documentos/30.pdf

39. Clark RH. The epidemiology of respiratory failure in neonates born at an estimated gestational age of 34 weeks or more. J Perinatol. 2005;25:251-7.

40. Flores-Compadre JL, Cruz F, Orozco G, Vélez A. Hipoxia perinatal y su impacto en el neurodesarrollo. Rev. chil. neuropsicol. (En línea), 2013;8:26-31.

41. Burke BL, Robbins JM, Hobbs CA, Clar Nesmith A, Tilford J. Trends in hospitalizations for neonatal jaundice and kernicterus in the United States, 1988-2005. Pediatrics. 2009;123:524-32.

42. Corujo-Santana C, Falcón-González JC, Borkoski-Barreiro SA, Pérez-Plasencia D, Ramos-Macías Á. Relación entre hiperbilirrubinemia neonatal e hipoacusia neurosensorial. Acta Otorrinolaringológica Española, 2015;66:326-331.

43. Wusthoff CJ, Loe IM. Impact of bilirubin-induced neurologic dysfunction on neurodevelopmental outcomes. Semin Fetal Neonatal Med. 2015; 20: 52-7.

44. Newman TB, Liljestrand P, Jeremy RJ, Ferriero DM, Wu YW, Hudes E, et al. Outcomes among newborns with total serum bilirubin levels of $25 \mathrm{mg}$ per deciliter or more. $\mathrm{N}$ Engl J Med. 2006;354:1889-1900.

45. Martínez JC. El real problema del recién nacido ictérico. Nuevas guías de la Academia Estadounidense de Pediatría. Arch Argent Pediatr. 2005;103:524-32.

46. Pérez RO, Lona JC, Quiles M, Verdugo MÁ, Ascencio $E P$, Benítez EA. Sepsis neonatal temprana, incidencia y factores de riesgo asociados en un hospital público del occidente de México. Revista chilena de infectología, 2015;32:447-452.

47. Valero K. Factores de riesgo para hipoacusia en recién nacidos que egresan de la unidad neonatal del Hospital Central de Maracay. Universidad de Carabobo. 2014. Tesina de Especialización en Neonatología. Universidad de Carabobo.

48. Als H. Toward a synactive theory of development: Promise for the assessment and support of infant individuality. Infant Mental Health Journal. 1982;3:229-43. 
49. Vericat A, Orden AB. Herramientas de screening del desarrollo psicomotor en Latinoamérica. Rev Chil Pediatr. 2010;81:391-401.

50. Lejarraga $H$, Menéndez AM, Menzano E, Guerra L, Biancato $S$, Pianelli $P$, et al. PRUNAPE: screening for psychomotor development problems at primary care level. Arch argent pediatr. 2008;106:119-25.

51. Casasbuenas, OL. Seguimiento neurológico del recién nacido pretérmino. Rev Neurol. 2005;40:65-7.

52. Ohlsson A, Jacobs SE. NIDCAP: A Systematic Review and meta-analyses of randomized controlled trials. Pediatrics. 2013;131:881-93.

53. Als H, Duffy FH, McAnulty GB, Fischer CB, Kosta S, Butler $\mathrm{SC}$, et al. Is the Newborn Individualized Developmental Care and Assessment Program (NIDCAP) effective for preterm infants with intrauterine growth restriction? J Perinatol. 2011;31:130-36.

54. Grivell R, Dodd J, Robinson J. The prevention and treatment of intrauterine growth restriction. Best Pract Res Clin Obstet Gynaecol 2009;23:795-807.

55. Bhutta ZA, Das JK, Bahl R, et al. Can available interventions end preventable deaths in mothers, newborn babies, and stillbirths, and at what cost? Lancet. 2014;384:347-70.

56. Penela-Vélez de Guevara MT, Gil-López SB, Martín-Puerto MJ, Romero-Escós MD, Herrera-Martín M, Urbón-Artero A. Estudio descriptivo de la asfixia perinatal y sus secuelas. Rev Neurol 2006;43:3-6.

57. Berglund $\mathrm{S}$, Grunewald $\mathrm{C}$, Pettersson $\mathrm{H}$, Cnattingius $\mathrm{S}$. Severe asphyxia due to delivery-related malpractice in Sweden 1990-2005. BJOG 2008;115:316-23.

58. Chang HH, Larson J, Blencowe $\mathrm{H}$, Spong $\mathrm{CY}$, Howson CP, CairnsSmith $\mathrm{S}$, et al. Born too soon preterm prevention analysis group. Preventing preterm births: analysis of trends and potential reductions with interventions in 39 countries with very high human development index. Lancet. 2013;381:223-34.

59. Gardosi J, Figueras F, Clausson B, Francis A. The customised growth potential: an international research tool to study the epidemiology of fetal growth. Paediatr Perinat Epidemiol. 2011;25:2-10.
60. Cordero Rodríguez Y, Peláez Puente M, De Miguel Abad M, Perales Santaella M, Barakat Carballo R ¿Puede el ejercicio físico moderado durante el embarazo actuar como un factor de prevención de la Diabetes Gestacional? Rev Int Cienc Deporte. 2012;8:1-5.

61. Weston EJ, Pondo T, Lewis MM, Martell-Cleary P, Morin C, Jewell B, et al. The burden of invasive early-onset neonatal sepsis in the United States, 2005-2008. Pediatr Infect Dis J. 2011;30:937-41.

62. Weissman SJ, Hansen NI, Zaterka-Baxter K, Higgins RD, Stoll BJ. Emergence of antibiotic resistance-associated clones among Escherichia coli recovered from newborns with early-onset sepsis and meningitis in the United States, 2008-2009. Journal of the Pediatric Infectious Diseases Society 2015;269-76.

63. Zaman K, Roy E, Arifeen SE, Rahman M, Raqib R, Wilson $E$, et al. Effectiveness of maternal influenza immunization in mothers and infants. N Engl J Med. 2008;359:1555-64.

64. Edmon K, Zaidi A. New approaches to preventing, diagnosing, and treating neonatal sepsis. PLoS Medicine. 2010;7:1-8.

65. Alderdice F, McCall E, Bailie C, Craig S, Dornan J, McMillen R, et al. Admission to neonatal intensive care with respiratory morbidity following 'term' elective caesarean section. Ir Med J. 2005;98:170-182.

66. Pardo Campos ML, Musso M, Keselman A, Gruñeiro L, Bergadá I, Chiesa A. Perfiles cognitivos en pacientes con hipotiroidismo congénito detectado y tratado en forma temprana. Archivos argentinos de pediatría. 2017;115:12-17.

67. Ramos Sánchez I, Márquez Luque A. Recién nacido de riesgo neurológico. Vox Paediatrica. 2000;8:5-10.

68. Romano Berindoague C, Macaya Ruiz A. Evolución neurológica, radiológia y cognitiva en niños nacidos con leucomalacia periventricular. Tesis Doctoral. Universidad Autónoma de Barcelona, 2014:1-73.

69. Vericat A, Orden AB. El desarrollo psicomotor y sus alteraciones: entre lo normal y lo patológico. Ciênc. Saúde Coletiva. 2013;18:2977-84. 